1 2

\title{
Reptation Quantum Monte Carlo Calculation of Charge Transfer: The Na-Cl Dimer
}

Yi Yao ${ }^{1}$ and Yosuke Kanai ${ }^{1,2, *}$

1. Department of Chemistry, University of North Carolina at Chapel Hill, Chapel Hill, North Carolina 27599

2. Condensed Matter and Materials Division, Lawrence Livermore National Laboratory, Livermore, California 94550

\section{ABSTRACT}

The phenomenon of ion pairing in aqueous solutions is of widespread importance in chemistry and physics, and charge transfer between the ions plays a significant role. We examine the performance of quantum Monte Carlo (QMC) calculations for describing the charge transfer behavior in a $\mathrm{NaCl}$ dimer. The influence of the fermion nodes is investigated by obtaining the electron density using the reptation Monte Carlo approach. The fermion nodes are given by single-particle orbitals in SlaterJastrow trial wavefunctions. We consider the single-particle orbitals from Hartree-Fock and density functional theory calculations with several exchange-correlation approximations. Appreciable dependence of the charge transfer on the fixed-node approximation was found although the total energy was found to be rather insensitive. Our work shows that a careful examination of the fixednode approximation is necessary for quantifying charge transfer in QMC calculations even when other properties such as reaction energetics are insensitive to the approximation.

*Email : ykanai@unc.edu 


\section{Introduction}

It is widely documented that many commonly-employed approximations to the exchange-correlation (XC) potential in density functional theory (DFT) calculation are inadequate for describing long-range charge $\operatorname{transfer}^{1,2}$ ENREF_2, for which the exact analytical behavior is $\mathrm{known}^{3}$. The short/intermediate range charge transfer behavior, however, has not been discussed to the same extent in the literature. This seemingly technical theoretical point actually has great implications for computer simulations of aqueous ionic solutions, which are crucial for developing various technological applications such as water desalination $^{4}$ and nano-fluidic devices ${ }^{5}$. Molecular dynamics (MD) simulations are extensively used to derive important scientific insights concerning behavior of ion pairs such as $\mathrm{NaCl}$ in aqueous systems ${ }^{6-}$ ${ }^{9}$ ENREF 7 . The pair correlation function of $\mathrm{Na}-\mathrm{Cl}$ separation distance (and hence the potential of mean force) has been shown to depend significantly on the underlying potential energy in the short/intermediate $\mathrm{Na}-\mathrm{Cl}$ separation distance of $2 \sim 6 \AA$ in $\mathrm{MD}$ simulations of $\mathrm{NaCl}$ in bulk water ${ }^{10}$, and developing a better empirical potential for such MD simulations would require an accurate description of the charge transfer between the ions. Therefore, accurate electronic structure calculations beyond DFT are desired for deriving important parameters for MD simulations ${ }^{11} 121314$ and also for assessing the performance of first-principles MD simulations based on DFT 15161718 .

At the same time, many high-level wavefunction-based electronic structure calculations such as coupledcluster theory ${ }^{19,20}$ do not scale well with the system size ${ }^{21}$, and applying these methods to aqueous systems containing hundreds of electrons is not practical today. Compared to these high-level wavefunction-based methods, quantum Monte Carlo (QMC) methods ${ }^{22-24}$ scale much more favorably with the system size. Having "embarrassingly-parallel" characteristic of the Monte Carlo algorithm, the QMC approaches are highly attractive, especially given the recent trend in high-performance computing to employ a large number of processing cores for achieving a better performance (more than 3 million cores in the fastest supercomputer in $2014^{25}$ ). Therefore, QMC approaches potentially present a highly attractive avenue for performing accurate electronic structure calculations of condensed phase systems such as a solvated $\mathrm{NaCl}$ in bulk water. Practical necessity to approximate nodes of the many-body wavefunction, however, makes QMC calculations inexact for Fermion systems despite the exchange and correlation effects being entirely taken into account. Although there exist approaches that avoid this approximation ${ }^{26-28}$, their applicability is presently still quite limited. This so-called fixed node approximation is often found accurate for obtaining reaction energetics 293031 . But, investigations into the influence of this fixed node approximation on electronic structure features such as charge transfer have been scarce ${ }^{32}$. As a first step toward accurate modeling of $\mathrm{NaCl}$ in water using QMC calculations, we investigated the influence of the fixed-node approximation on describing charge transfer in a $\mathrm{NaCl}$ dimer in the short/intermediate range by using reptation Monte Carlo approach ${ }^{33}$.

\section{Computational Methods}

The exact value of the charge, $q$, on $\mathrm{Na}^{+\mathrm{q}}-\mathrm{Cl}^{-\mathrm{q}}$ depends on the theoretical approach that is used to partition the electron density between $\mathrm{Na}$ and $\mathrm{Cl}$ atoms; we employed Bader analysis ${ }^{34}$ that is based on locating the boundaries that satisfy the condition:

$$
\nabla(r)=0
$$

where $(r)$ is the electron density, and the gradient is taken with respect to the three-dimensional coordinate $r{ }^{35}$ This approach is particularly appealing because the electron density can be obtained from QMC calculations using reptation Monte Carlo approach ${ }^{33}$ as discussed later.

Hartree-Fock, DFT, MP2, and CCSD calculations were performed using Gaussian basis as implemented in Gaussian09 code ${ }^{36}$. Def2-TZVPPD ${ }^{37}$ Gaussian basis set is used for these calculations. CRENBL effective core potentials ${ }^{38}$ are used to take into account the core electrons. For Na, semi-core electrons ( $2 \mathrm{~s}$ and $2 \mathrm{p}$ states) are explicitly treated. Plane-wave basis set was also employed for HF and DFT 


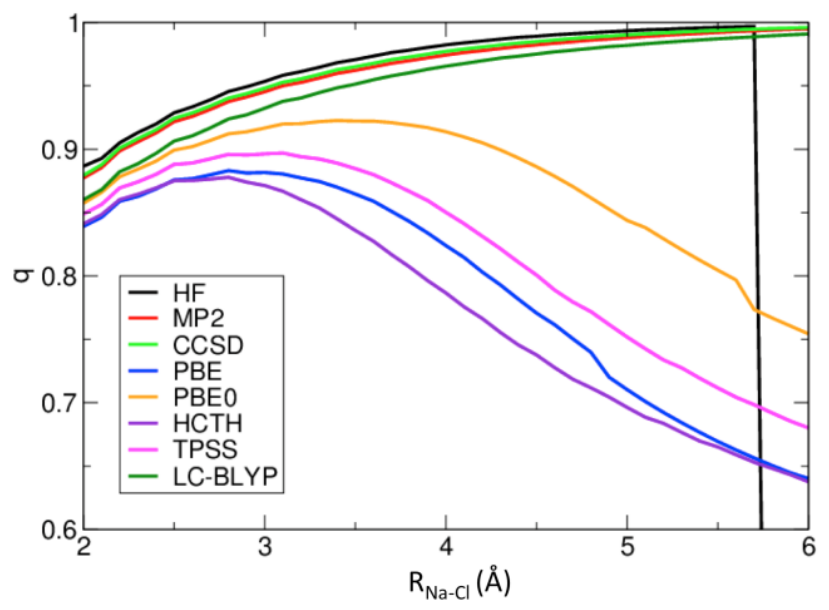

Figure 1: Amount of charge transfer between $\mathrm{Na}$ and $\mathrm{Cl}$ atoms as a function of the separation distance. Bader analysis was use for the charge partitioning. All the calculations here are performed using Gaussian basis set, and the comparison to plane-wave basis set in given in Fig S-1 in Supplementary Information.

calculations for comparison, and they were performed using CPMD code ${ }^{39}$, Troullier-Martin normconserving pseudopotentials ${ }^{40}$ are used for plane-wave calculations. The semi-core electrons for $\mathrm{Na}$ are treated explicitly. Plane-wave basis set is used with a kinetic energy cutoff of 80 Ry. Reptation quantum Monte Carlo calculations were performed using QWalk code ${ }^{41}$ with Slater-Jastrow trial wavefunctions ${ }^{29}$.

\section{Results and Discussion}

The antisymmetric fermion nodes in the QMC calculations here are determined by Slater-Jastrow trial wavefunctions, and single-particle orbitals from density functional theory (DFT) calculations are used to construct the Slater determinant. Therefore, the performance of DFT calculations with various exchangecorrelation (XC) approximations is discussed first. The importance of non-local electron-electron exchange interaction is largely responsible for the difficulty in using DFT to calculate charge transfer behavior accurately using common XC functionals. Self-interaction error in XC functionals, which is largely responsible as the primary source of error for charge transfer ${ }^{42}$, is widely discussed as one of the greatest challenges in DFT today, and it appears that a perfect remedy does not yet exist. Comprehensive reviews on the topic are available in the literature (See, for example, Ref. ${ }^{12}$ for recent reviews). XC functionals with Hartree-Fock (HF) exchange have become popular in recent years because they appear to improve a wide range of properties in various systems from molecules to solids ${ }^{43}$. A physical basis for mixing $\mathrm{HF}$ exact exchange in XC functional of DFT is based on the adiabatic connection formula ${ }^{44}$. In addition to those hybrid functionals that include a fixed fraction of $\mathrm{HF}$ exchange (e.g. PBE ${ }^{45}$ ), another class of hybrid XC functionals relies on separating the exchange functional into the short-range and long-range parts by using the error function ${ }^{46,47}$. The long-range part is described by HF exchange integral through

$$
E_{X}^{L C}=\frac{1}{2} \underbrace{}_{i}{ }_{j}^{\text {occ occ }} \quad{ }_{i}^{*}\left(r_{1}\right)_{j}^{*}\left(r_{2}\right) \frac{\operatorname{erf}\left(r_{12}\right)}{r_{12}}{ }_{j}\left(r_{1}\right)_{i}\left(r_{2}\right) d^{3} r_{1} d^{3} r_{2}
$$

where $\quad j$ is $j$-th $\sigma$-spin single-particle orbital and $\eta$ is a parameter that determines the long and short range separation. For instance, $\eta$ is set to 0.33 bohr $^{-1}$ in the popular range-separated LC-BLYP approximation ${ }^{48}$. The range separated $\mathrm{XC}$ functionals has been found to perform remarkably well in describing charge transfer behavior $^{49}$.

Charge transfer in an ionic dimer abruptly vanishes at the critical separation distance (at which point the charges on atoms are zero, exhibiting a quantum phase transition behavior ${ }^{52}$ ) in the electronic ground state. This critical distance is given by

$R_{c}=e^{2}\left(I_{+} A\right)^{1}$ 
where $I_{+}$is the ionization potential of the less electronegative atom and $A_{\text {. }}$ is the election affinity of the more electronegative atom ${ }^{3}$, and this critical separation distance is approximately $9.4 \AA$ for a NaCl dimer. This long-range charge transfer behavior is not well described by most commonly-employed $\mathrm{XC}$ functionals in DFT calculations, and consequently the behavior in the short/intermediate $\mathrm{Na}-\mathrm{Cl}$ separation distance of 2 6 $\AA$ is also influenced. Figure 1 shows the charge, $q\left(\right.$ on $\left.\mathrm{Na}^{+\mathrm{q}}-\mathrm{Cl}^{-\mathrm{q}}\right)$ as a function of $\mathrm{Na}-\mathrm{Cl}$ separation distance for DFT calculations and other popular wave-function theory calculations. $\mathrm{CCSD}^{50}$ and $\mathrm{MP} 2^{51}$ calculations both show a similar behavior of a monotonic increase, and HF calculation follows them closely except that the critical separation distance is located quite early at $5.7 \AA^{52}$. DFT calculations with the generalized gradient approximation (GGA) for the $\mathrm{XC}$ functional exhibits qualitatively incorrect behavior in both $\mathrm{PBE}^{53}$ and $\mathrm{HCTH}^{54}$ forms such that a well defined maximum is seen at the separation distance of $3 \sim 4 \AA$ before it decays monotonically. Note that $q$ does not plateau to $0 e$ (neutral atoms) in the dissociation limit but to 0.41 $e$ for both PBE and HTCH functionals as previously discussed in Ref. ${ }^{52}$ (See Fig S-3 in Supplementary Information). Although hybrid-GGA XC approximation, $\mathrm{PBE} 0^{45}$, appears to improve the behavior slightly such that the maximum is located farther out, the qualitative erroneous behavior remains the same as for the GGA XC approximations. Also, meta-GGA XC approximation, TPSS ${ }^{55}$, exhibits the similar behavior, being closer to the GGA XC approximations than to the hybrid-GGA XC approximation ${ }^{52}$. The range-separated functional, LC-BLYP performs remarkably well, following closely the CCSD and MP2 charge transfer behavior. These findings are independent of using plane-waves or Gaussian functions as the basis set as long as the basis set employed is large enough (See Figure S-2 in Supplementary Information).

With recent computational and methodological advancement, it has become possible to compute the electron density rigorously within QMC calculation using reptation Monte Carlo (RMC) approach ${ }^{33}{ }^{56}$. We employed RMC approach here to obtain the electron density of a $\mathrm{NaCl}$ dimer. Unlike the popular diffusion Monte Carlo approach ${ }^{57}$, RMC approach allows us to calculate the expectation value of operators that do not commute with Hamiltonian (e.g. the density) by having access to the "pure" distribution instead of the mixed distribution $^{23}$. In RMC calculations, the random walk is performed with the Green's function $G$ of the imaginary-time Schrodinger equation ${ }^{22}$ in the "path" space $l=\left[R_{0}, R_{1}, . ., R_{n}\right]$ where $R$ denotes the many-electron coordinates. Sampling the path distribution,

$$
(l)={ }_{T}\left(R_{0}\right) G\left(R_{0}, R_{1}, \quad \ldots G\left(R_{n 1}, R_{n}, \quad{ }_{T}\left(R_{n}\right),\right.\right.
$$

where $\tau$ is the imaginary time, and $\quad{ }_{T}$ is the trial wavefunction. The pure distribution is obtained at $R_{n / 2}$ for calculating the electronic density. In the present calculations, a path length of 3 a.u. and a time step of 0.01 a.u. are used. The remaining physical approximation for obtaining accurate electron density through solving many-body Schrodinger equation is the fixed node approximation, which makes the many-body wavefunction nodes the same as that of the trial wavefunction. The trial wavefunction here is obtained using a variational Monte Carlo calculation ${ }^{58}$ from Slater determinant of single-particle orbitals from DFT or HF calculations, multiplied by a two-body Jastrow correlation factor; the variational parameters, including electron-electron, electron-nucleus, and electron-electron-nucleus terms, are optimized by variance minimization $^{59,60}$ ENREF 42. To examine the influence of the fixed node approximation, several Slater determinants are obtained using Kohn-Sham (KS) single-particle orbitals from DFT calculations with different XC approximations. In particular, we employed the single-particle orbitals from HF and DFT calculations with the range-separated XC (i.e. LC-BLYP) and PBE0 XC with a varying fraction of HF exchange $^{61}$ (from 0 to 1 with an increment of 0.25 ) for the Slater determinant part of the trail wavefunction. The variational principle allows us to assess different fermion node approximations by computing the total energies ${ }^{61}$. 


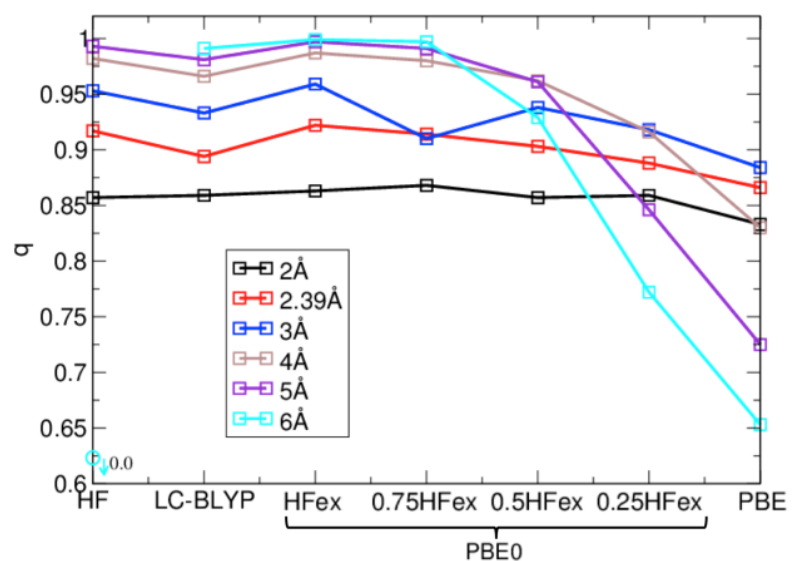

Figure 2: Charge transfer between $\mathrm{Na}$ and $\mathrm{Cl}$ atoms for different $\mathrm{Na}-\mathrm{Cl}$ separation distances in $\mathrm{HF}$ and DFT calculations with various $X C$ approximations.

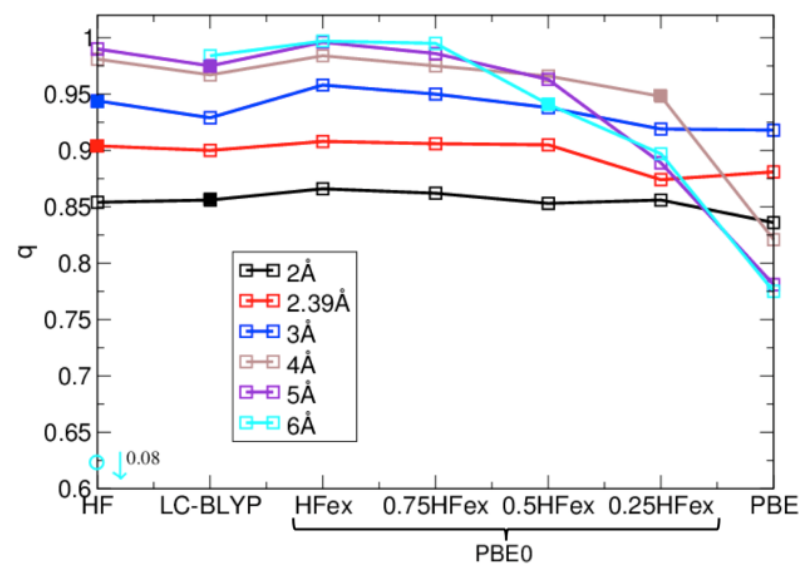

Figure 3: Charge transfer between $\mathrm{Na}$ and $\mathrm{Cl}$ atoms for different $\mathrm{Na}-\mathrm{Cl}$ separation distances in RMC calculations with the fermion nodes from DFT calculations with various $\mathrm{XC}$ approximations. The filled squares indicate the fermion node that yields the lowest enerav.

We first calculated the charge transfer with the DFT calculations as shown in Figure 2 for comparison. Close to the equilibrium separation distance of $2.39 \AA$ (CCSD value), all the calculations give a similar value of $0.9 e$ for the charge transfer. However, as the separation distance increases, the charge transfer behavior becomes increasingly more dependent of the XC approximation (Figure 2). Figure 3 shows the dependence of the charge transfer in RMC calculation on the approximated fermion nodes that are given by the different sets of the single-particle orbitals. In general, we observe a similar trend as in the DFT calculations; the RMC calculations show less charge transfer when less HF exchange fraction is used in DFT to obtain the single-particle orbitals for the fermion nodes. Comparing Figures 2 and 3, we observe that the dependence on the fermion node for RMC calculation is, however, not as significant as the dependence of DFT calculation on the XC approximation although not negligible. For instance, the RMC calculation shows the charge transfer of $0.78 e$ at the separation distance of $6 \AA$ using the fermion nodes from PBE singleparticle orbitals (no HF exchange) while the DFT-PBE calculation itself yields $0.65 e$ at the same separation distance. Except for the case with the fermion nodes from PBE single-particle orbitals (no HF exchange mixing), the dependence of RMC calculation on the fermion nodes generally appears to be modest, resulting in the maximum variation in the charge transfer of at most $0.1 e$ (for the separation distance of $6 \AA$ ). Using

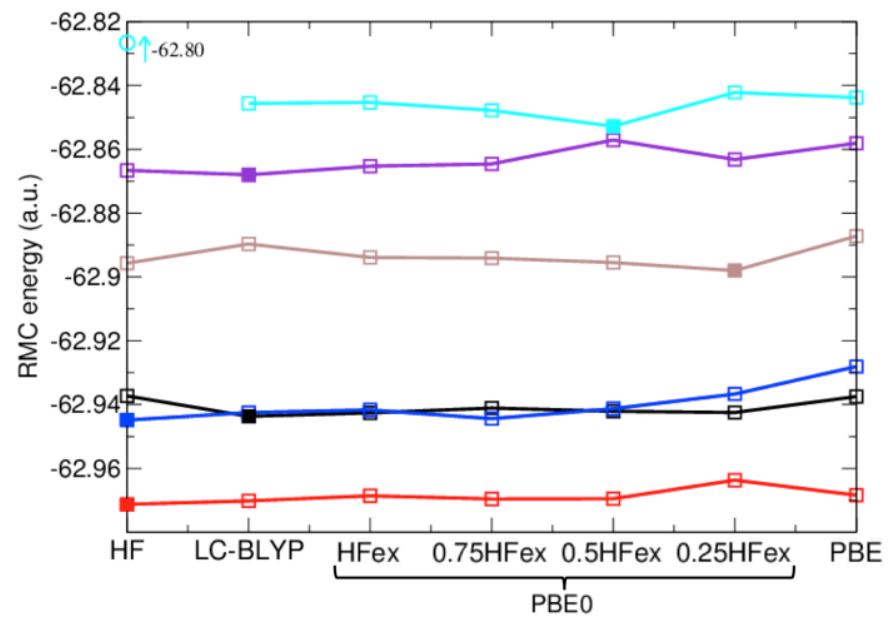

Figure 4: Absolute total energies from RMC calculations with fermion nodes from HF and DFT calculations with various XC approximations for different $\mathrm{Na}-\mathrm{Cl}$ separation distances. The filled squares indicate the fermion node that yields the lowest energy.

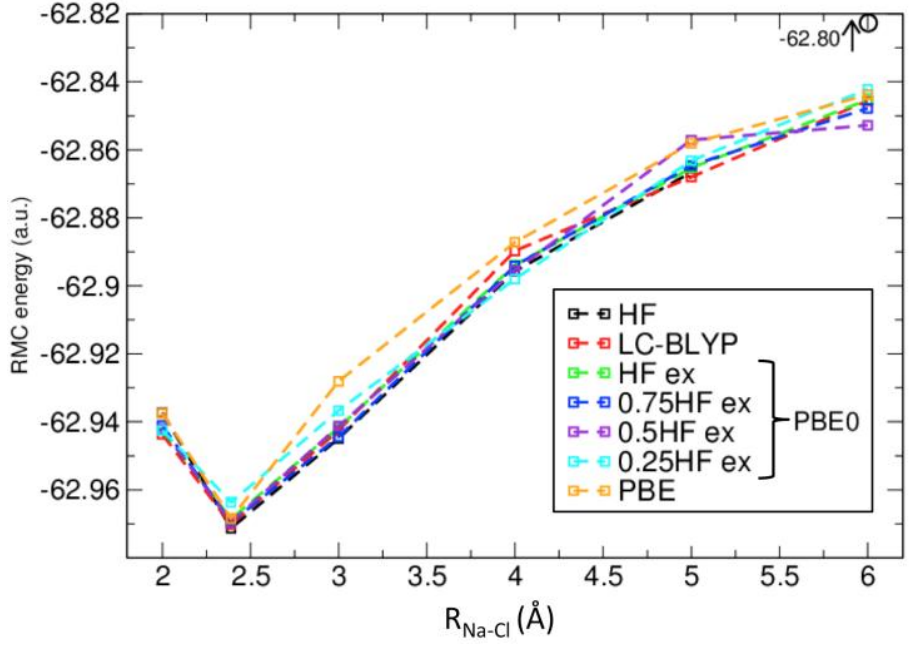

Figure 5: Absolute total energy change as a function of $\mathrm{Na}-\mathrm{Cl}$ separation distance in RMC calculations with fermion nodes from HF and DFT calculations with various XC approximations for different $\mathrm{Na}-\mathrm{Cl}$ separation distances. 

calculation is similar to the DFT calculation with LC-BLYP XC approximation. In the case of using HF single-particle orbitals for the fermion nodes, the RMC calculation yields the similar charge transfer behavior as the LC-BLYP case except that no charge transfer is predicted for the separation distance of $6 \AA$ (see Figure 3) because of the erroneous critical separation distance of $5.4 \AA$ in HF calculation.

The filled squares in Figure 3 indicate the fermion node that yields the lowest total energy for a given separation distance in RMC calculation. Absolute total energies from the RMC calculations are shown in Figure 4., and the statistical uncertainties are within 0.0002 a.u.. We were not able to find an obvious relation between the $\mathrm{Na}-\mathrm{Cl}$ separation distance and the $\mathrm{HF}$ exchange mixing for the best fermion node in the RMC calculations. Figure 4 also shows that the dependence of the total energy on the fermion node is quite small with the variation of $\sim 0.01$ a.u. when it is compared to the total energy change (Figure 5) in the $\mathrm{Na}-\mathrm{Cl}$ separation distance of 2 6 $\AA$. CCSD calculation yields the total energy change of 0.116 a.u. in going from the equilibrium separation distance of $2.39 \AA$ to $6 \AA$ while RMC values range from 0.117 a.u. (fermion nodes from $0.5 \mathrm{HF}$ Ex in PBE0) to 0.125 a.u. (fermion nodes from LC-BLYP). Overall, the total energy change is found rather insensitive to the fixed node approximation even though the charge transfer varies as much as $0.2 e$ depending on the fixed nodes.

\section{Conclusion}

In summary, we used the reptation Monte Carlo (RMC) approach to investigate the influence of the fixed fermion node approximation in quantum Monte Carlo (QMC) calculations of short/intermediate-range charge transfer in a $\mathrm{Na}-\mathrm{Cl}$ dimer. The fermion nodes were approximated using a Slater determinant of singleparticle orbitals from density functional theory (DFT) calculations, and the performance of DFT calculations with various exchange (XC) approximations was discussed. We observe that the hybrid, meta-GGA, and GGA XC approximations (PBE, HCTH, PBE0, TPSS) do not follow the charge transfer behavior in CCSD and MP2 calculations for $\mathrm{Na}-\mathrm{Cl}$ separation distance of $2 \sim 6 \AA$, which is particularly important for investigating $\mathrm{NaCl}$ in water. The charge transfer behavior calculated via the Hartree-Fock (HF) calculation and by using the range-separated LC-BLYP approximation, however, follows closely to the monotonic increase observed in CCSD and MP2 calculations. In light of these observations, we used the RMC approach to calculate the electron density in QMC calculations where the fermion node approximation was used with Slater determinants of the single-particle orbitals from HF, LC-BLYP and a series of hybrid XC functionals (PBE0) with varying amount of HF exchange. We observed a considerable dependence of the charge transfer on the fermion node approximation. Depending on the HF mixing, the amount of charge transfer varies as much as $0.2 e$. This dependence is not as large as the XC dependence of DFT calculations (as much as $0.4 e$ ). At the same time, we observe that the total energy in QMC calculations was found rather insensitive to the fixed node approximation. These results indicate that a careful examination of the fixed node approximation is necessary for quantifying charge transfer in QMC calculations, while other ground state properties, such as reaction energies remain rather insensitive to the approximation (e.g. See Ref. ${ }^{31}$ ). Finally, the appreciable dependence of charge transfer also suggests that the fixed node approximation needs to be carefully examined also for reaction energetics if the dipole contribution dominates the total energy changes in charge transfer reactions.

\section{Acknowledgement}

We gratefully acknowledge support by the donors of the Petroleum Research Fund, Grant 52494-DNI6 and National Energy Research Computing Center, which is supported by the Office of Science of the U.S. Department of Energy under Contract No. DE-AC02-05CH11231 for computational resources. We thank Lucas K. Wagner (UIUC) for helpful discussions.

\section{Reference}


(1) Mori-Sanchez, P.; Cohen, A. J.: The derivative discontinuity of the exchange-correlation functional. Physical Chemistry Chemical Physics 2014, 16, 14378-14387.

(2) Cohen, A. J.; Mori-Sánchez, P.; Yang, W.: Challenges for Density Functional Theory. Chemical Reviews 2011, 112, 289-320.

(3) Perdew, J. P.; Parr, R. G.; Levy, M.; Balduz, J. L.: Density-Functional Theory for Fractional Particle Number: Derivative Discontinuities of the Energy. Physical Review Letters 1982, 49, 1691-1694.

(4) Humplik, T.; Lee, J.; O’Hern, S. C.; Fellman, B. A.; Baig, M. A.; Hassan, S. F.; Atieh, M. A.; Rahman, F.; Laoui, T.; Karnik, R.; Wang, E. N.: Nanostructured materials for water desalination. Nanotechnology 2011, 22, 292001.

(5) Fornasiero, F.; Park, H. G.; Holt, J. K.; Stadermann, M.; Grigoropoulos, C. P.; Noy, A.; Bakajin, O.: Ion exclusion by sub-2-nm carbon nanotube pores. Proceedings of the National Academy of Sciences 2008, 105, 17250-17255.

(6) Lin, S.; Buehler, M. J.: Mechanics and molecular filtration performance of graphyne nanoweb membranes for selective water purification. Nanoscale 2013, 5, 11801-11807.

(7) Liu, L.; Chen, X.: Fast Ion Transport and Phase Separation in a Mechanically Driven Flow of Electrolytes through Tortuous Sub-Nanometer Nanochannels. ChemPhysChem 2013, 14, 24132418.

(8) Cohen-Tanugi, D.; Grossman, J. C.: Water Desalination across Nanoporous Graphene. Nano Letters 2012, 12, 3602-3608.

(9) Cohen-Tanugi, D.; Grossman, J. C.: Water permeability of nanoporous graphene at realistic pressures for reverse osmosis desalination. The Journal of Chemical Physics 2014, 141, - .

(10) Timko, J.; Bucher, D.; Kuyucak, S.: Dissociation of $\mathrm{NaCl}$ in water from ab initio molecular dynamics simulations. The Journal of Chemical Physics 2010, 132, -.

(11) Smith, D. E.; Dang, L. X.: Computer simulations of $\mathrm{NaCl}$ association in polarizable water. The Journal of Chemical Physics 1994, 100, 3757-3766.

(12) Kim, J. S.; Wu, Z.; Morrow, A. R.; Yethiraj, A.; Yethiraj, A.: Self-Diffusion and Viscosity in Electrolyte Solutions. The Journal of Physical Chemistry B 2012, 116, 12007-12013.

(13) Luo, Y.; Jiang, W.; Yu, H.; MacKerell, A. D.; Roux, B.: Simulation study of ion pairing in concentrated aqueous salt solutions with a polarizable force field. Faraday Discussions 2013, 160, 135-149.

(14) Yu, H.; Whitfield, T. W.; Harder, E.; Lamoureux, G.; Vorobyov, I.; Anisimov, V. M.; MacKerell Jr, A. D.; Roux, B.: Simulating monovalent and divalent ions in aqueous solution using a Drude polarizable force field. Journal of Chemical Theory and Computation 2010, 6, 774-786.

(15) Timko, J.; Bucher, D.; Kuyucak, S.: Dissociation of $\mathrm{NaCl}$ in water from ab initio molecular dynamics simulations. The Journal of Chemical Physics 2010, 132, 114510.

(16) Gaiduk, A. P.; Zhang, C.; Gygi, F.; Galli, G.: Structural and electronic properties of aqueous $\mathrm{NaCl}$ solutions from ab initio molecular dynamics simulations with hybrid density functionals. Chem. Phys. Lett. 2014, 604, 89-96.

(17) Ding, Y.; Hassanali, A. A.; Parrinello, M.: Anomalous water diffusion in salt solutions. Proceedings of the National Academy of Sciences 2014, 111, 3310-3315.

(18) Scipioni, R.; Schmidt, D. A.; Boero, M.: A first principles investigation of water dipole moment in a defective continuous hydrogen bond network. The Journal of Chemical Physics 2009, 130, $-$

(19) Bartlett, R. J.; Musiał, M.: Coupled-cluster theory in quantum chemistry. Reviews of Modern Physics 2007, 79, 291-352.

(20) Lyakh, D. I.; Musiał, M.; Lotrich, V. F.; Bartlett, R. J.: Multireference Nature of Chemistry: The Coupled-Cluster View. Chemical Reviews 2011, 112, 182-243.

(21) Head-Gordon, M.; Artacho, E.: Chemistry on the computer. Physics Today 2008, 61, 58. 
(22) Foulkes, W. M. C.; Mitas, L.; Needs, R. J.; Rajagopal, G.: Quantum Monte Carlo simulations of solids. Reviews of Modern Physics 2001, 73, 33-83.

(23) Austin, B. M.; Zubarev, D. Y.; Lester, W. A.: Quantum Monte Carlo and Related Approaches. Chemical Reviews 2011, 112, 263-288.

(24) Towler, M. D.: The quantum Monte Carlo method. physica status solidi (b) 2006, 243, 2573-2598.

(25) Top500.org, 2014.

(26) Tubman, N. M.; DuBois, J. L.; Hood, R. Q.; Alder, B. J.: Prospects for release-node quantum Monte Carlo. The Journal of Chemical Physics 2011, 135, 184109-4.

(27) Umrigar, C. J.; Toulouse, J.; Filippi, C.; Sorella, S.; Hennig, R. G.: Alleviation of the Fermion-Sign Problem by Optimization of Many-Body Wave Functions. Physical Review Letters 2007, 98, 110201.

(28) Booth, G. H.; Thom, A. J. W.; Alavi, A.: Fermion Monte Carlo without fixed nodes: A game of life, death, and annihilation in Slater determinant space. The Journal of Chemical Physics 2009, $131,-$.

(29) Wagner, L. K.: Quantum Monte Carlo for Ab Initio calculations of energy-relevant materials. International Journal of Quantum Chemistry 2014, 114, 94-101.

(30) Kanai, Y.; Grossman, J. C.: Role of exchange in density-functional theory for weakly interacting systems: Quantum Monte Carlo analysis of electron density and interaction energy. Physical Review A 2009, 80, 032504.

(31) Wu, Z.; Allendorf, M. D.; Grossman, J. C.: Quantum Monte Carlo Simulation of Nanoscale MgH2 Cluster Thermodynamics. Journal of the American Chemical Society 2009, 131, 13918-13919.

(32) Lucas, K. W.: Transition metal oxides using quantum Monte Carlo. Journal of Physics: Condensed Matter 2007, 19, 343201.

(33) Baroni, S.; Moroni, S.: Reptation Quantum Monte Carlo: A Method for Unbiased Ground-State Averages and Imaginary-Time Correlations. Physical Review Letters 1999, 82, 47454748.

(34) Bader, R.: Atoms in Molecules - A Quantum Theory; Oxford University Press, 1990.

(35) Henkelman, G.; Arnaldsson, A.; Jonsson, H.: A fast and robust algorithm for Bader decomposition of charge density. Comp Mater Sci 2006, 36, 354-360.

(36) Frisch, M.; Trucks, G.; Schlegel, H. B.; Scuseria, G.; Robb, M.; Cheeseman, J.; Scalmani, G.; Barone, V.; Mennucci, B.; Petersson, G.: Gaussian 09, Revision A. 02, Gaussian. Inc., Wallingford, CT 2009, 200.

(37) Rappoport, D.; Furche, F.: Property-optimized Gaussian basis sets for molecular response calculations. The Journal of Chemical Physics 2010, 133, -.

(38) Hurley, M. M.; Pacios, L. F.; Christiansen, P. A.; Ross, R. B.; Ermler, W. C.: Abinitio relativistic effective potentials with spin - orbit operators. II. K through Kr. The Journal of Chemical Physics 1986, 84, 6840-6853.

(39) Hutter, J.; Ballone, P.; Bernasconi, M.; Focher, P.; Fois, E.; Goedecker, S.; Parrinello, M.; Tuckerman, M.: CPMD Version 3.0. Max-Planck Institut f ur Festk orperforschung and IBM Zurich Research Laboratory 1995, 1999.

(40) Troullier, N.; Martins, J. L.: Efficient pseudopotentials for plane-wave calculations. Physical Review B 1991, 43, 1993-2006.

(41) Wagner, L. K.; Bajdich, M.; Mitas, L.: QWalk: A quantum Monte Carlo program for electronic structure. Journal of Computational Physics 2009, 228, 3390-3404.

(42) Zhao, Y.; Truhlar, D. G.: Density Functional for Spectroscopy: No Long-Range SelfInteraction Error, Good Performance for Rydberg and Charge-Transfer States, and Better Performance 
on Average than B3LYP for Ground States. The Journal of Physical Chemistry A 2006, 110, 1312613130 .

(43) Burke, K.: Perspective on density functional theory. The Journal of Chemical Physics 2012, 136, -.

(44) Parr, R. G.; Yang, W.: Density Functional Theory of Atoms and Molecules; Oxford University Press, 1994.

(45) Perdew, J. P.; Ernzerhof, M.; Burke, K.: Rationale for mixing exact exchange with density functional approximations. The Journal of Chemical Physics 1996, 105, 9982-9985.

(46) Iikura, H.; Tsuneda, T.; Yanai, T.; Hirao, K.: A long-range correction scheme for generalized-gradient-approximation exchange functionals. The Journal of Chemical Physics 2001, 115, 3540-3544.

(47) Leininger, T.; Stoll, H.; Werner, H.-J.; Savin, A.: Combining long-range configuration interaction with short-range density functionals. Chem. Phys. Lett. 1997, 275, 151-160.

(48) Tawada, Y.; Tsuneda, T.; Yanagisawa, S.; Yanai, T.; Hirao, K.: A long-range-corrected time-dependent density functional theory. The Journal of Chemical Physics 2004, 120, 8425-8433.

(49) Vydrov, O. A.; Scuseria, G. E.: Assessment of a long-range corrected hybrid functional. The Journal of Chemical Physics 2006, 125, -.

(50) Purvis, G. D.; Bartlett, R. J.: A full coupled - cluster singles and doubles model: The inclusion of disconnected triples. The Journal of Chemical Physics 1982, 76, 1910-1918.

(51) Møller, C.; Plesset, M. S.: Note on an Approximation Treatment for Many-Electron Systems. Physical Review 1934, 46, 618-622.

(52) Ruzsinszky, A.; Perdew, J. P.; Csonka, G. I.; Vydrov, O. A.; Scuseria, G. E.: Spurious fractional charge on dissociated atoms: Pervasive and resilient self-interaction error of common density functionals. The Journal of Chemical Physics 2006, 125, - .

(53) Perdew, J. P.; Burke, K.; Ernzerhof, M.: Generalized Gradient Approximation Made Simple. Physical Review Letters 1996, 77, 3865-3868.

(54) Hamprecht, F. A.; Cohen, A. J.; Tozer, D. J.; Handy, N. C.: Development and assessment of new exchange-correlation functionals. The Journal of Chemical Physics 1998, 109, 6264-6271.

(55) Tao, J.; Perdew, J. P.; Staroverov, V. N.; Scuseria, G. E.: Climbing the Density Functional Ladder: Nonempirical Meta-Generalized Gradient Approximation Designed for Molecules and Solids. Physical Review Letters 2003, 91, 146401.

(56) Pierleoni, C.; Ceperley, D. M.: Computational Methods in Coupled Electron-Ion Monte Carlo Simulations. ChemPhysChem 2005, 6, 1872-1878.

(57) Anderson, J. B.: A random-walk simulation of the Schr[o-umlaut]dinger equation: $\mathrm{H}$ [sup + ][sub 3]. The Journal of Chemical Physics 1975, 63, 1499-1503.

(58) Metropolis, N.; Rosenbluth, A. W.; Rosenbluth, M. N.; Teller, A. H.; Teller, E.: Equation of State Calculations by Fast Computing Machines. The Journal of Chemical Physics 1953, 21, 10871092.

(59) Umrigar, C. J.; Wilson, K. G.; Wilkins, J. W.: Optimized trial wave functions for quantum Monte Carlo calculations. Physical Review Letters 1988, 60, 1719-1722.

(60) Umrigar, C. J.; Filippi, C.: Energy and Variance Optimization of Many-Body Wave Functions. Physical Review Letters 2005, 94, 150201.

(61) Wagner, L.; Mitas, L.: A quantum Monte Carlo study of electron correlation in transition metal oxygen molecules. Chem. Phys. Lett. 2003, 370, 412-417. 


\section{Cover Figure}

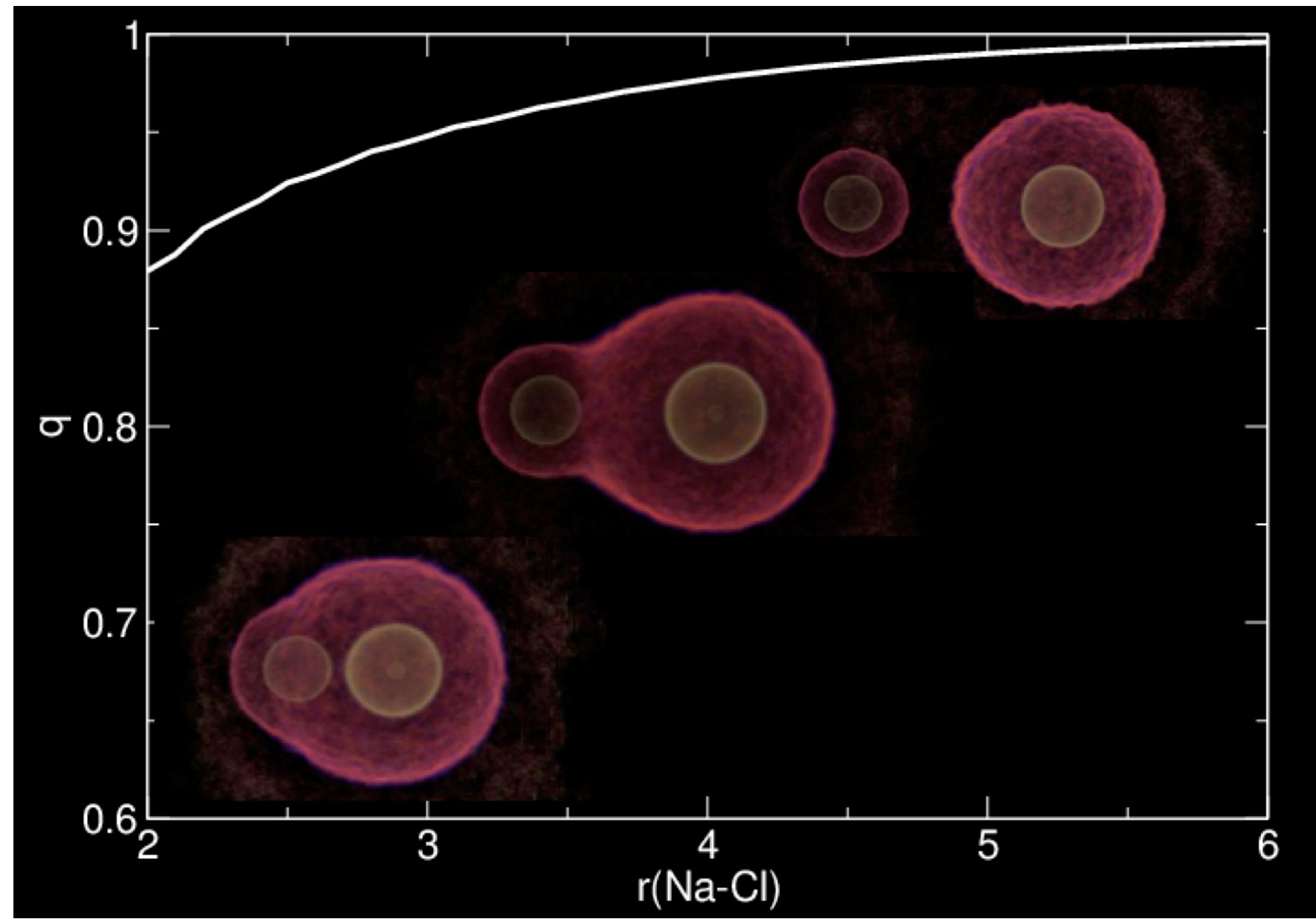

45

46

47

48

49

50

51

52

53

54

55

56

57

58

59

60

61

62

63

64

65 


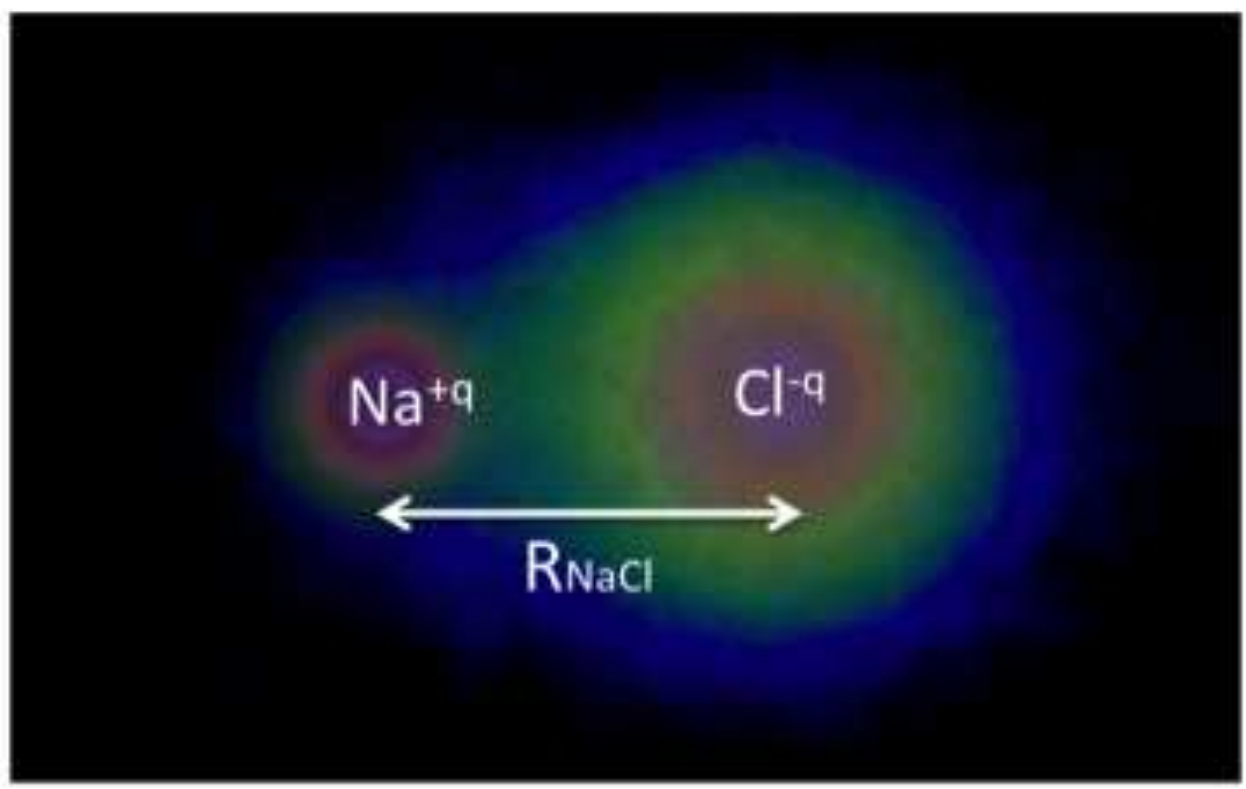

\section{$\because 2 A$}

अ $2.39 A$

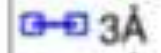

$0 \rightarrow 4 A$

$\rightarrow-5 A$

6-6A
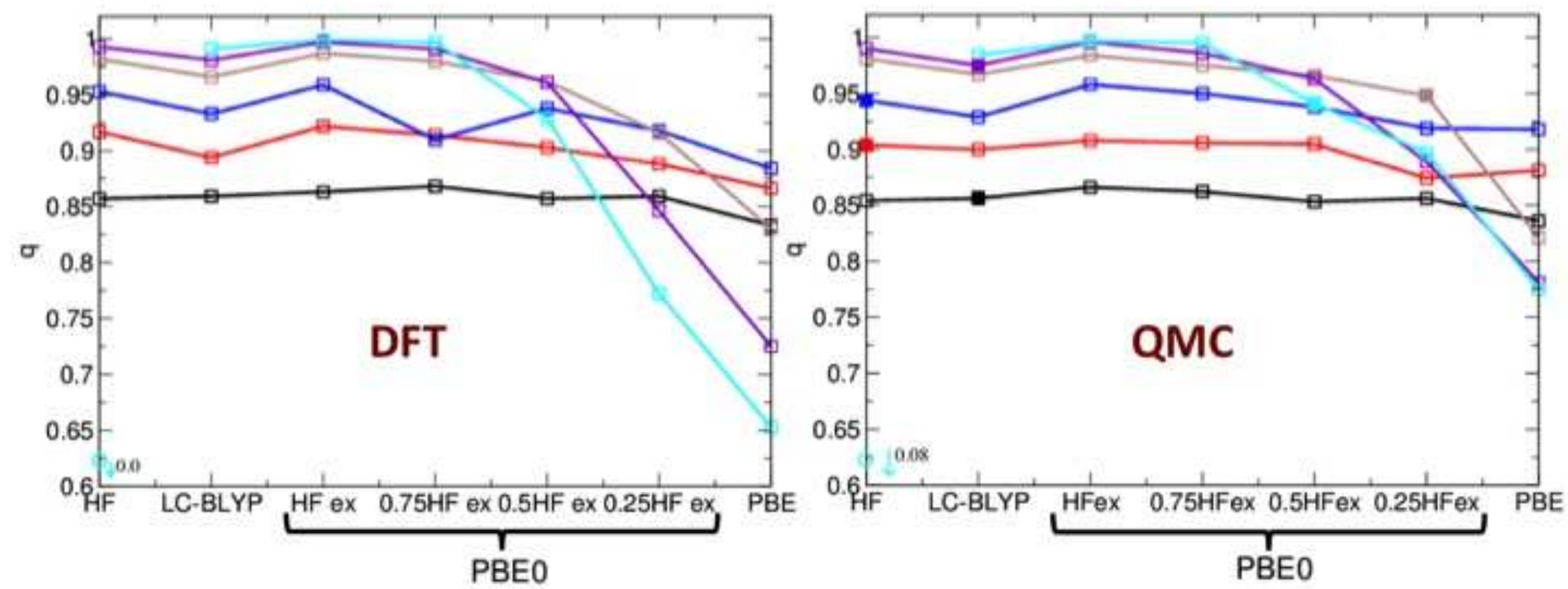
7

\section{Author Photos}

Yi Yao

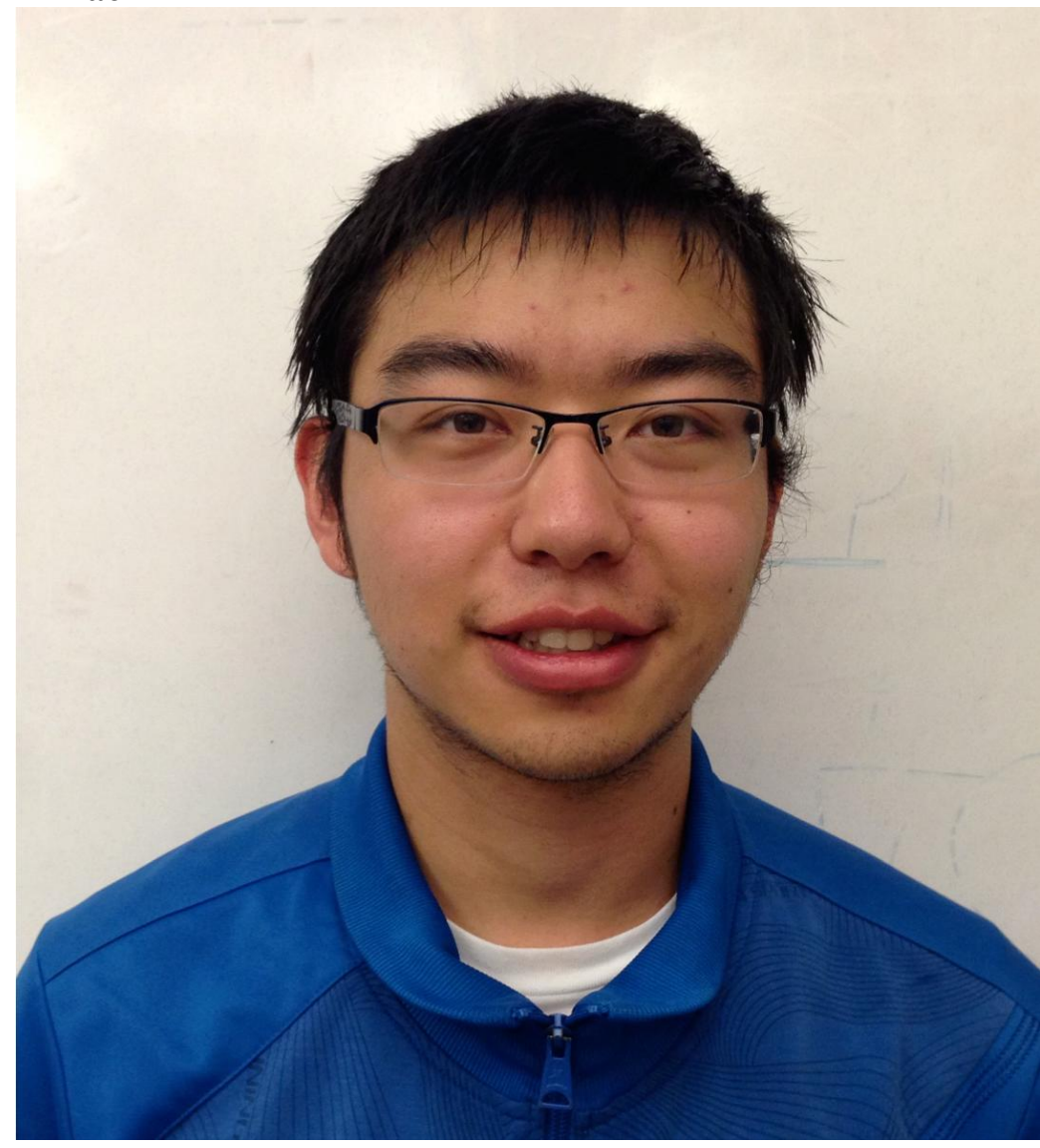

(1)

.




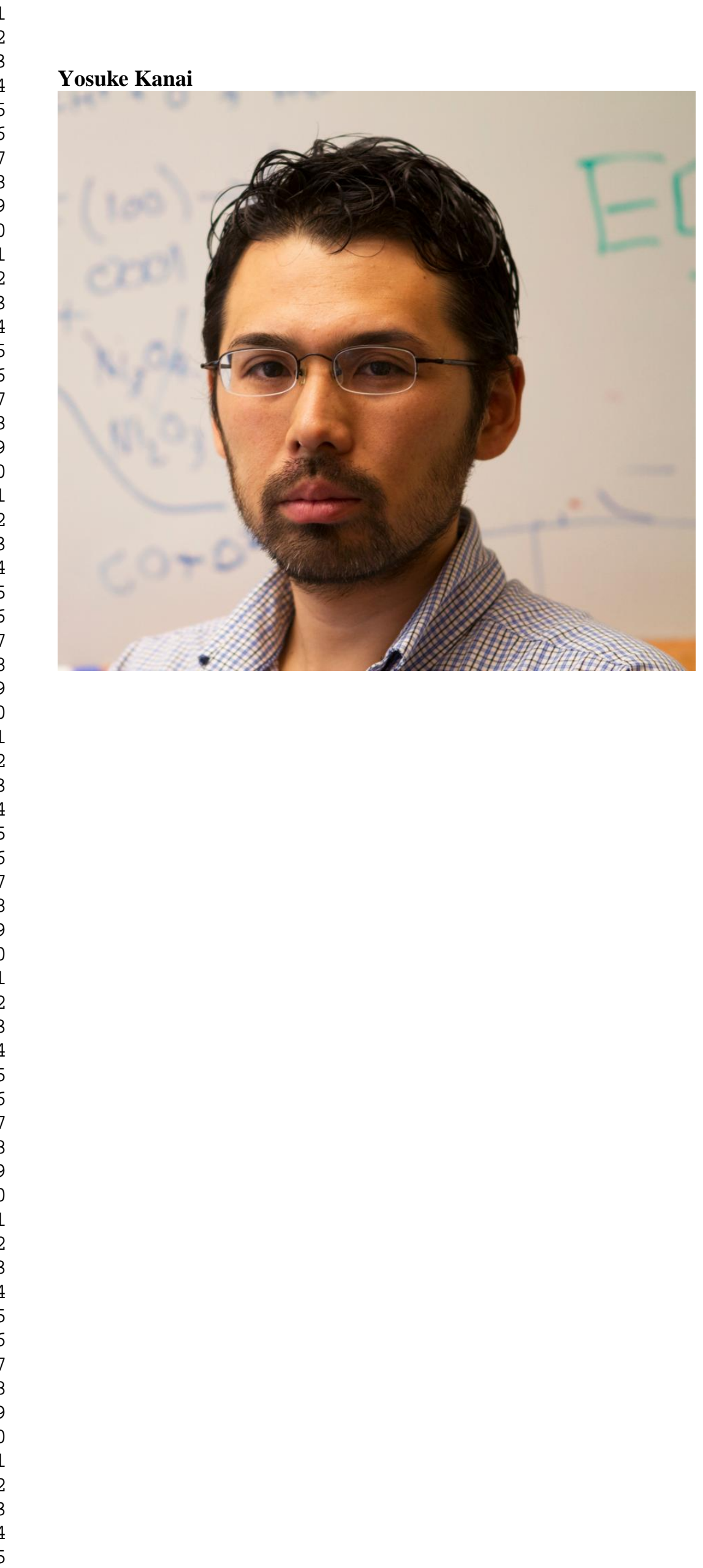


Yi Yao obtained his B.S. in Chemical Physics from University of Science and Technology of China in 2012. He is currently a Ph.D. student working with Prof. Yosuke Kanai and Prof. Max Berkowitz in Department of Chemistry at the University of North Carolina at Chapel Hill. His Ph.D. research is on computational simulations of aqueous ionic solutions.

Yosuke Kanai is an Assistant Professor in Department of Chemistry at the University of North Carolina at Chapel Hill and a visiting scientist in Condensed Matter and Materials Division at Lawrence Livermore National Laboratory (LLNL). He holds a Ph.D. in Theoretical Chemistry from Princeton University, and he was a Berkeley Nanoscience and Nanoengineering Institute Post-doctoral Scholar at the University of California at Berkeley. He subsequently worked at LLNL as a Lawrence Fellow prior to joining the University of North Carolina at Chapel Hill in 2011. 
Click here to download high resolution image

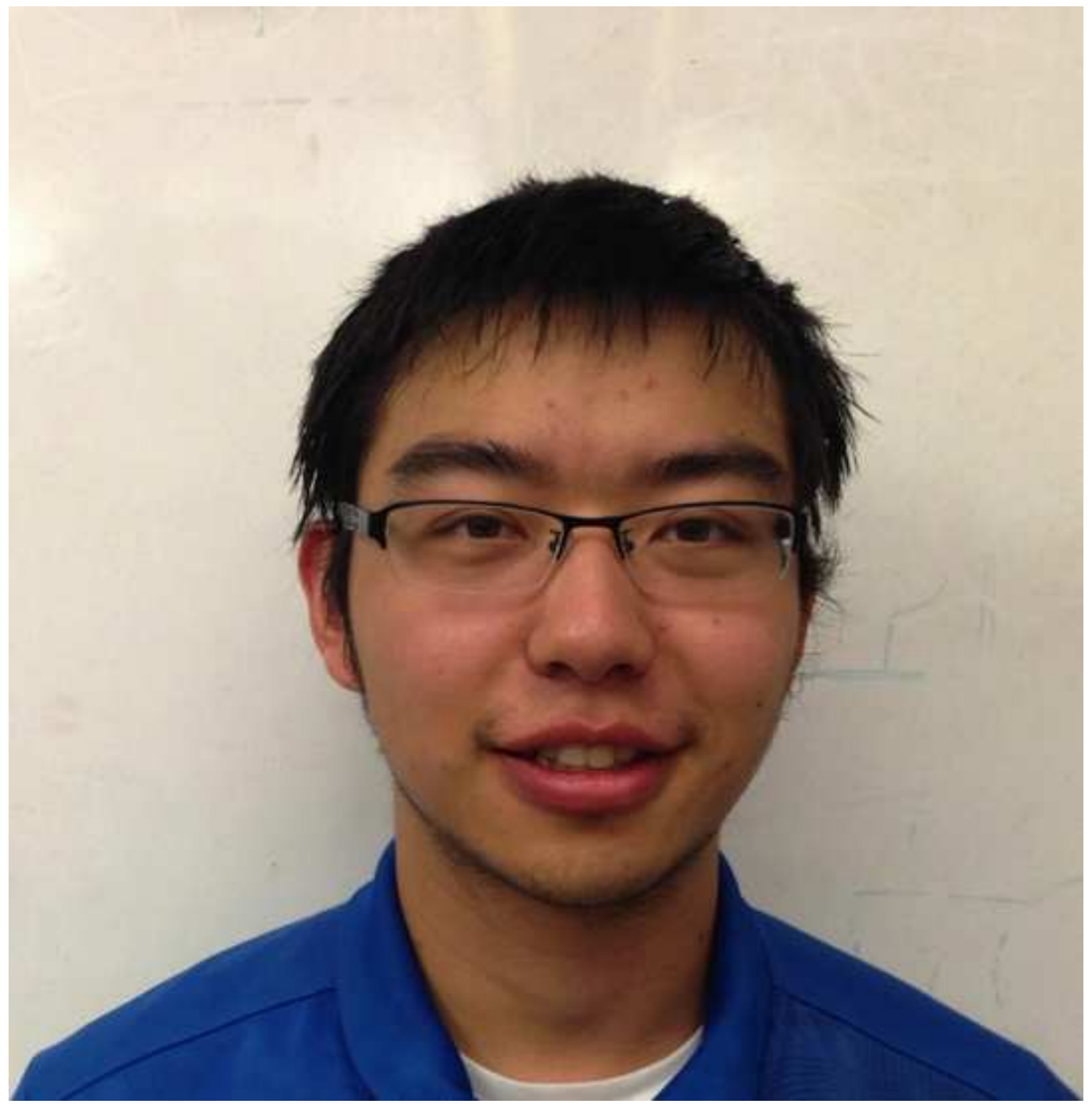


Click here to download high resolution image

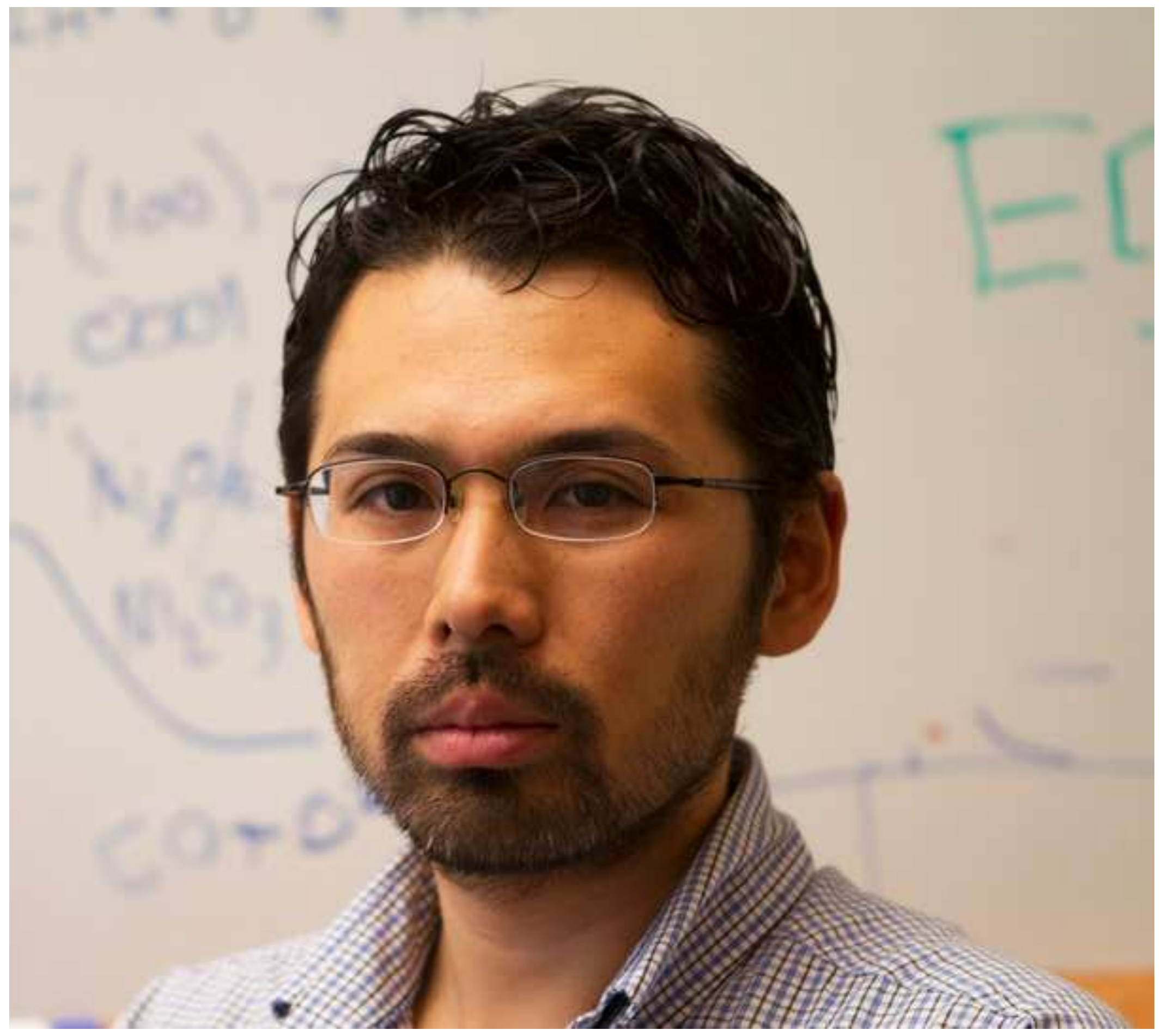


Click here to download high resolution image

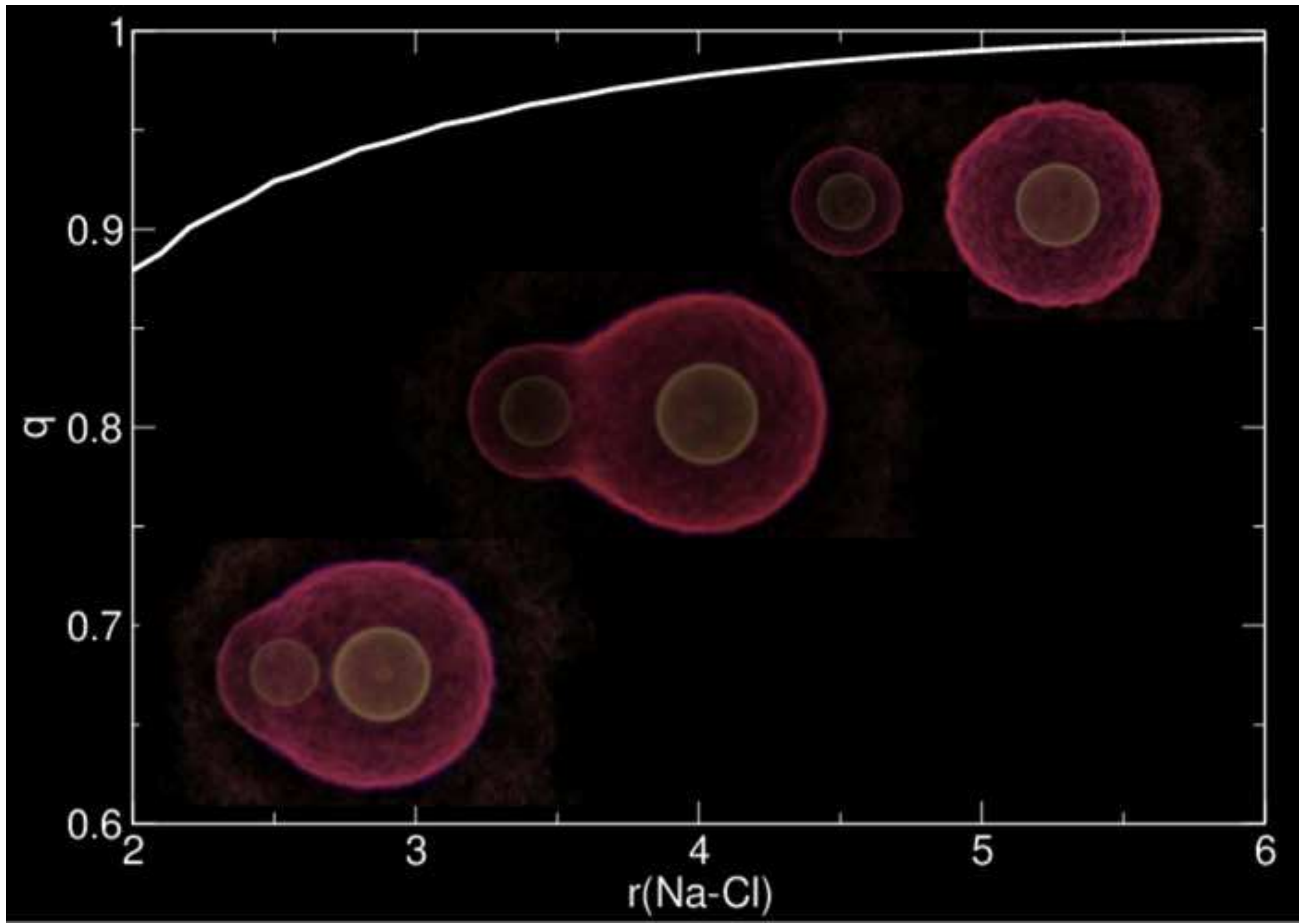

